\title{
New twist for anthropic principle
}

\section{The originator of the doctrine that our observations of the Universe are biased because they are made by us now concludes that there have been at most two critical steps in biological evolution.}

THE anthropic principle is Dr Brandon Carter's choice of a philosophical middle ground between what he calls the "primitive anthropocentrism" of the preCopernicans and "its equally unjustifiable antithesis" of extreme representations of the relativistic cosmological principle, the assertion that, "apart from local inhomogeneities", no place (or time) in the Universe can be privileged in any way. When Carter introduced his principle a decade ago (IAU Symp. 63, 291; 1974), his objective was modestly to point out that the mere fact that our observations of the Universe are made by people who are the products of some 3,000 million years of biological evolution implies an inescapable bias. Carter's latest version of the argument, now published as part of the report of last year's meeting at the Royal Society on the constants of physics (Phil. Trans. R. Soc. A 310, 347-363; 1983) is more radical, and a challenge to biologists as well as astronomers.

The reasons why the anthropic principle should figure in a symposium on the constants of physics are not as surprising as they may seem. The luminosity of a star of given mass, and thus the speed of its evolution from a young star to, say, a red giant, depends linearly on the value of the gravitational constant. If this value were only a little greater than it is now thought to be, the Sun would have run through its evolution faster, so that biological evolution might have got no further than, say, the dinosaurs before the Earth was engulfed by a red giant. And a smaller gravitational constant might never have allowed for the particular circumstances that led to the emergence of living things in the first place. So there is a chance of learning something about the value of the gravitational constant simply by exploiting the circumstance that its evaluation depends on observations made by people. Carter now says that if he had known that the name "anthropic principle" would be so widely used, he would have looked harder for a different way of referring to the bias caused by self-selection.

In passing, Carter directs well-aimed darts at two fashionable preoccupations, speculation about extraterrestrial life and Dirac's large-numbers hypothesis, each of which he says is sustained by neglect of the anthropic principle. Telescoped, the argument is this. The search for extraterrestrial life is based on the hypothesis that the emergence of living things is likely whenever geophysical circumstances are favourable, and thus on a denial of the contradictory hypothesis that the emergence of life is improbable. But, says Carter, the only evidence bearing on the question is the observation (by ourselves) that life has indeed emerged on the surface of the Earth, whence there is no way of distinguishing between the two hypotheses.

Dirac's large-numbers hypothesis is logically identical. Dirac at the outset argued that coincidence between the age of the Universe (strictly, the Hubble time) and the gravitational constant is so remarkable that one must expect the gravitational constant to decrease with time so as to preserve the coincidence. (Numerically, the Hubble time is $10^{58}$ in units in which the velocity of light, Planck's constant and the gravitational coupling strength are determined by the three-halves power of the square of the proton mass, of the order of $10^{38}$ in the same units.) Carter's view is that the coincidence is not a coincidence at all, but a consequence of the selection bias occasioned because the observations are necessarily made by people whose existence requires a Hubble time large enough so that some stars can have completed their evolution and have generated the chemical elements of which people are made, but not so long that all stars have run their course.

This is the stuff of lunch-room arguments about the anthropic principle in the past decade. Carter's new departure begins with another coincidence - that between the time-span of biological evolution on the surface of the Earth (say, $0.4 \times 10^{10}$ years) and the length of time the Sun is likely to spend as a hydrogen-burning star (say $10^{10}$ years) before becoming a red giant with dimensions engulfing the Earth's orbit.

What connection can there be between two such apparently independent quantities as the timescale of biological evolution, presumably determined by the complexity of living organisms, and the timescale of stellar evolution, determined (in Carter's words) by "the weakness of gravitation"? None, he says. The apparent coincidence is merely a consequence of the "habitual mistake" of overlooking the anthropic principle.

The most plausible way of accounting for it is to suppose that the timescale of biological evolution, itself a Markovian process (one thing leading to another) whose course must be uncertain, is on the average much longer than the time allowed by the lifespan of the Sun as a hydrogenburning star. So the observation, after a mere 4,000 million years, that there are sentient beings able to contemplate their own emergence is a sign that, in the event, the course of evolution on the Earth has been exceptionally short.

Carter takes this argument one argumentative step further. Suppose, he says, that the evolution of sentient beings depends on $n$ critical steps (defined as unlikely evolutionary steps, which are thus "slow", compared with the majority of evolutionary steps). Then it is possible to show that the chance of all these $n$ steps being completed by time $t$ is proportional to $t^{n}$ and also to show that evolution (the Sun's time as a hydrogen-burning star or $T_{0}$ ) exceeds that occupied by evolution so far by a quantity of the order of $(1 / n) t$. But this time is itself of the order of $T_{0}$, whence Carter concludes that $n$ must be a whole number greater than zero and less than or equal to two - in short, either 1 or 2 . Carter insists that the general character of this result does not depend on the details of the calculation but acknowledges that it poses a dilemma: how can the fossil record be squared with the conclusion that there have been at most two critical steps in evolution so far? His own preference is to face the dilemma head on; he recommends the evolution of the genetic code and the organization of the nervous system as candidates for the two critical steps.

What weight should be given to this conclusion? In his paper, Carter does not seek to mollify his critics, dismissing the attention being paid to the "inflationary universe" in all its varieties as an attempt to resurrect the "beguiling notion" of the perfect cosmological principle, and complaining that those who hold that only falsifiable hypotheses are admissible are in effect saying that science has accomplished nothing so far.

The Popper school will be further offended that Carter not merely uses the verb "to induce" but that he openly uses the language of Bayesian probability, the calculus of informed guesswork as some consider it. Even his friends may say that he has too lightly discarded the possibility that his coincidence may be explained if evolution is a rapid process which happens on the Earth to have been slow. But Carter is plainly not afraid to stir up trouble and has also provided a tangible example of how the anthropic principle may be made quantitative.

John Maddox 